\title{
The role of S-bond in tenoxicam keto-enolic tautomerization
}

\author{
Sergey G. Arkhipov ${ }^{1,2}$, Peter S. Sherin ${ }^{1,3}$, Alexey S. Kiryutin ${ }^{1,3}$, Vladimir A. Lazarenko ${ }^{4}$, Christian Tantardini ${ }^{5}$ \\ ${ }^{I}$ Novosibirsk State University, Novosibirsk, Russian Federation; \\ ${ }^{2}$ Boreskov Institute of Catalysis SB RAS; \\ ${ }^{3}$ International Tomography Center; \\ ${ }^{4}$ National Research Center "Kurchatov Institute"; \\ ${ }^{5}$ Center for Energy Science and Technology, Skoltech Skolkovo Institute of Science and Technology; \\ arksergey@gmail.com
}

The tenoxicam (4-hydroxy-2-methyl-N-2-pyridyl-2H-thieno(2,3-e)-1,2-thiazine-3-carboxamide 1,1-dioxide), is a non-steroidal antiinflammatory drug (NSAID), member of oxicam family, widely used in the treatment of osteoporosis. Tenoxicam (TXM) could be present in the $\beta$-keto-enolic form (BKE) or $\beta$-diketone (BDK) and in a zwitterionic form (ZWC) (Figure 1). However, in solid form (more than 20 different compounds including polymorphic modifications, co-crystals, and solvates) [1 and present work] TXM has predominantly found in the zwitterionic form (ZWC). While in a dissolved form, keto-enolic equilibrium is observed since recorded by us experimental absorption and fluorescence spectra for various TXM solutions show presence two forms of TXM (called A and B) in solvents with high polarity and only $\mathbf{A}$ form of TNX in low polar solvents (cyclohexane, toluene, chloroform, dioxane). This led us to think about the possibility to obtain solid forms of tenoxicam contain it in BKE or BDK form.

Figure 1 TXM molecular structures of three different forms: zwitterionic (ZWC), left; $\beta$-keto-enolic (BKE), center; $\beta$-diketone (BDK), right. Structural differences between forms are highlighted by red.

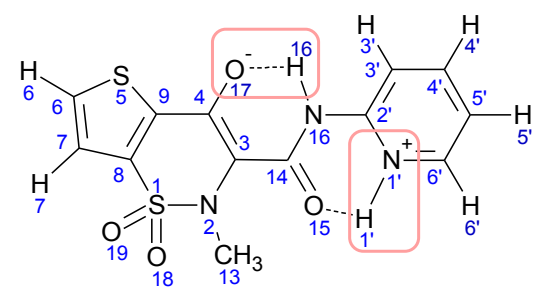

ZWC

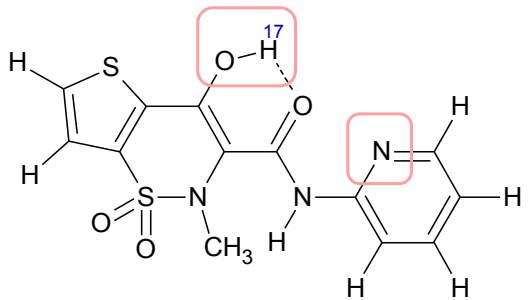

BKE

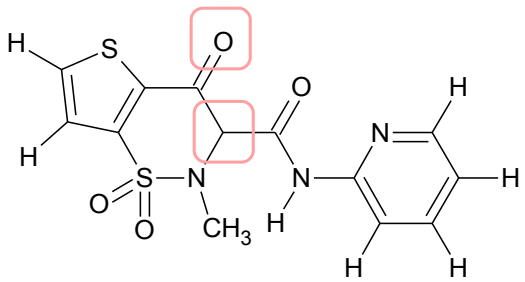

BDK

A set of NMR measurements using various 1D- and 2D- techniques were used to assign which of TXM keto-enolic form (see Figure 1) belong to the $\mathbf{A}$ and $\mathbf{B}$ forms observed in a liquid environment. As a result, form $\mathbf{A}$ observed by optical methods assigns to $\mathbf{B K E}$ form and the form $\mathbf{B}-$ to $\mathbf{Z W C}$. ${ }^{1} \mathrm{H}$ NMR spectra of tenoxicam in $\mathrm{CDCl}_{3}$ detected at various temperatures from -55 to $25{ }^{\circ} \mathrm{C}$ show almost $100 \%$ TXM in form of BKE at $25^{\circ} \mathrm{C}$ and almost $100 \%$ TXM in $\mathbf{Z W C}$ form at $-55^{\circ} \mathrm{C}$.

Taking into account optical and NMR data about the domination of BKE form in low polar solvents at room temperature, we tried to obtain solid forms of TNX containing TXM in BKE form by its crystallization from cyclohexane, toluene, dioxane, and chloroform. These experiments showed no crystal phase from cyclohexane and powder of TXM polymorph I from dioxane and toluene.

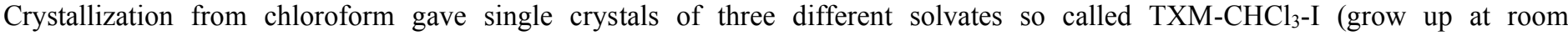
temperature), $\mathrm{TXM}-\mathrm{CHCl}_{3}$-II (grow up at $-18^{\circ} \mathrm{C}$ ) and $\mathrm{TXM}-\mathrm{CHCl}_{3}$-III (grow up at $-18^{\circ} \mathrm{C}$ ). But in all these solvates $\mathrm{TXM}$ presents in ZWC form.

For understanding why TNX exists in BKE form in solution, but crystallize in ZWC form, DFT calculations in vacuo were made. It shows that BKE to be the most thermodynamically stable form, ZWC is less stable and BDK is the least stable $(\Delta \mathrm{G}$ between BKE and these two forms of $2.20 \mathrm{kcal} / \mathrm{mol}$ and $12.49 \mathrm{kcal} / \mathrm{mol}$, respectively). But BKE form is characterized by a large twist between A 2pyridyl ring and TXM backbone with respect to almost flat ZWC form. Planarization of BKE form diminishes the energy difference between flatten BKE and ZWC forms almost to $0.15 \mathrm{kcal} / \mathrm{mol}$ that indicates a presence of another thin interaction within TXM molecule predisposing it to crystallization in ZWC form. This thin interaction was showed to be S-bond between thiophenil ring and carbonyl oxygen according to the analysis of intramolecular interactions within natural bond orbital theory [4]. This S-bond is significantly stronger for ZWC form as compared with flatten BKE form and it should be considered as the driving force of TXM crystallization 
[1] J. R. Patel, R. A. Carlton, T. E. Needham, C. O. Chichester and F. G. Vogt, Int. J. Pharm., 2012, 436, 685 —706.

[2] R. G. Cantera, M. G. Leza and C. M. Bachiller, J. Pharm. Sci., 2002, 91 , $2240-2251$

[3] G. Bolla , P. Sanphui and A. Nangia , Cryst. Growth Des., 2013, 13 , 1988 - 2003

[4] B. R. Beno, K.-S. Yeung, M. D. Bartberger, L. D. Pennington and N. A. Meanwell , J. Med. Chem., 2015, 58, 4383 - 4438

\section{Keywords: tenoxicam, solvates, keto-enolic tautomerization}

The authors would like to thank Dr. Anatoly A. Politov for useful discussion. SGA would like to thank Prof. Dr. Elena V. Boldyreva for her ongoing support. CT would like to thank his former supervisor Prof. Dr. Elena V. Boldyreva and his present supervisor Prof. Dr. Artem R. Oganov for their ongoing support.

SGA is indebted to Ministry of Science and Higher Education of the Russian Federation (project AAAA-A19-119020890025-3). PSS and ASK thank Ministry of Science and Higher Education of the Russian Federation for access to optical and NMR equipment (AAAAA16-116121510087-5). 